\title{
Development of an e-health app to support women prescribed adjuvant endocrine therapy after treatment for breast cancer
}

This article was published in the following Dove Press journal:

Patient Preference and Adherence

Jo Brett

Mary Boulton

Eila Watson

Faculty of Health and Life Sciences, Oxford Brookes University, Oxford OX3 OFL, UK
Correspondence: Jo Brett

Faculty of Health and Life Sciences,

Oxford Brookes University, Jack Straw's

Lane, Marston, Oxford OX3 OFL, UK

Tel +44I865 482696

Email jbrett@brookes.ac.uk
Background: Adjuvant endocrine therapy (AET) is prescribed to women for 5-10 years after treatment for estrogen receptor positive $(\mathrm{ER}+\mathrm{ve})$, early-stage breast cancer. AET has proven effectiveness in reducing the risk of recurrence of breast cancer and mortality. However, adherence is known to be suboptimal with around $20 \%$ discontinuing by 2 years and up to $50 \%$ discontinuing by 5 years. Interventions are needed to support women taking AET after breast cancer. The aim of this study was to develop and pilot test an e-health app for this population.

Methods: Two focus groups $(\mathrm{n}=15)$ and five interviews were conducted with women following treatment for early-stage breast cancer to assess the likely acceptability of an e-health app and to inform the content (Phase I). Following development of a prototype e-health app, a simple heuristic usability test was completed by five women in order to identify any design usability problems (Phase II). A further 18 women used the app for 1 month between July and August 2016, after which they were interviewed by telephone to collect their experiences and views of the app (Phase III).

Results: The prototype e-health app included evidence-based information on effectiveness of AET, an electronic side-effects diary, a peer support forum, a repeat prescription reminder, suggested strategies for facilitating adherence and managing any side effects that occur, and a link to further evidence and useful organizations for further information and support. The app was received positively by women. Women found the app useful as it emphasized the importance of taking AET, helped them manage their side effects and provided details of support organizations, while offering empathy and exchange of suggestions for self-management strategies through the peer support forum.

Conclusion: Overall, findings suggest that this novel e-health app has potential as a feasible medium for promoting adherence to AET. Future research should evaluate the efficacy of the app in supporting women and promoting adherence.

Keywords: adjuvant endocrine therapy, breast cancer, e-health app, support

\section{Plain language summary}

Around eight out of ten women are diagnosed with breast cancer triggered by estrogen, and prescribed adjuvant endocrine therapy which blocks the estrogen in the body. This medication is taken for up to 10 years, but some women do not continue taking the medication for this amount of time, due to side effects of the drug, lack of information and lack of support.

We developed an e health app for smart phones, tablets and computers, to inform and support women taking AET. The app was developed with the help of 20 women who have had early stage breast cancer, which informed the content of the app: information about AET, about how to get support, evidence of how effective AET is, an electronic side effects diary to record side effects, an online forum where women can chat with each other, and a prescription reminder. 
We asked 18 women taking AET to try the app for one month, then give us feedback on the app. The women were very positive about the app and found all the sections useful.

The app now needs to be tested in a bigger population to find out whether it helps increase adherence to AET.

\section{Introduction}

Adjuvant endocrine therapies (AETs) such as tamoxifen and aromatase inhibitors have proven clinical benefits in reducing the risk of recurrence of breast cancer when taken for 5-10 years, transforming outcomes for women with estrogen receptor-positive (ER+ve) breast cancer. ${ }^{1}$ However, despite these recognized benefits, there is good evidence that adherence to AET is poor: around $20 \%$ of those prescribed AET have discontinued treatment by 2 years and up to $50 \%$ by 5 years. ${ }^{7,8}$ Non-adherence to AET, whether through poor compliance or treatment cessation, is associated with a twoto three-fold increased risk of breast cancer recurrence and mortality. ${ }^{2-6}$

Factors shown to contribute to sub-optimal adherence include significant side effects, psychological problems, lack of information and support, poor patient-clinician relationships, poor recall, and limited belief in the efficacy of, or concerns about the medication. ${ }^{7-12}$ With shorter hospital follow-up times, a focus on more personalized treatment and reduced use of chemotherapy, ${ }^{14}$ it is increasingly important to support women with breast cancer to adhere to AET, to protect against recurrence and mortality. ${ }^{13-15}$ Innovative models of community-based follow-up care are therefore needed to inform, support and motivate adherence to AET.

E-health interventions, including smartphone and other e-health technologies, have seen enormous growth internationally over the past decade, changing the shape of health care and clinical research. ${ }^{16}$ Smartphones and tablets have become useful tools in the practice of evidence-based medicine at the point of care, and increasingly play a role in patient education, disease self-management and remote monitoring of patients. Use of e-health may also help integrate self-management support into aftercare for cancer patients. ${ }^{15}$ Support for self-management is particularly important for cancer survivors as health professionals have limited resources to provide such support and evidence suggests that cancer survivors want to be involved in health care decisions. ${ }^{17,18}$ Since patient-initiated aftercare is becoming more common, integrating technology-based self-management support into a single platform will provide cancer survivors with the knowledge, skills and confidence to manage the long-term implications of cancer treatment. ${ }^{19}$ There are a growing number of contemporary e-health apps that provide educational and self-assessment tools for women with breast cancer, but to date no app has been developed specifically to educate and support women taking long-term AET. ${ }^{20-23}$ We aimed to explore, develop and pilot a patientcentered e-health app to support and motivate women taking AET. We report here the patient-led development of the app (Phase I), usability of the prototype app (Phase II), and feasibility of the prototype app (Phase III).

\section{Methods}

A qualitative approach was adopted to inform the development and feasibility of a prototype app. The study was approved by the Oxford Brookes University Ethics committee. All participants gave their written informed consent prior to participation in the study, and it was conducted in accordance with the Declaration of Helsinki.

\section{Sample and recruitment}

Women who had completed active treatment for early-stage breast cancer (1-111A), were 18 years or older and able to provide informed consent were eligible for the study. Recruitment was through the UK charity Breast Cancer Care's and Independent Patient Cancer Voices' patient forums and newsletters. Participants were recruited between January 2016 and March 2016 for the development phase of the study and in June and July 2016 for the pilot of the prototype. For the pilot phase, women participating needed to own a smartphone, tablet or computer.

\section{Design and setting}

Phase I: informing the development of the app

Two focus groups $(n=15)$ and five telephone interviews were conducted to gain women's perspective on the acceptability of using an app to provide support to women prescribed AET and promote adherence. Possible content of the app was also explored. Focus groups took place at a charity headquarters and cancer center in the South East of England and lasted up to 1 hour. Telephone interviews were conducted with women who wanted to participate, but were not able to attend the focus groups. The focus groups and interviews were facilitated by an experienced qualitative health care researcher (JB).

Following the analysis of the focus groups and the interviews, a prototype app was developed by an app developer, 
informed by the data from Phase I. This was an iterative process over a 3-month period. Researchers, service users and the app developer worked collaboratively throughout this process. The app was an independent app, with restricted access for the purposes of this development phase.

\section{Phase II: testing the usability of the app}

Five women from Phase I participated in a simple heuristic usability test of the app to identify any design usability problems. ${ }^{24}$ It has been shown by Nielsen in $1994^{25}$ that three to five users are sufficient to uncover most usability problems. Usability assessments were conducted at the participant's home under the supervision of the researcher (JB). The following tasks were performed: Task 1: download the app; Task 2: navigate between the sections; Task 3: record side effects of AET experienced in the past week on electronic side-effect diary reporting the severity of the side effects (mild, moderate or severe); Task 4: set a repeat prescription reminder; and Task 5: access online forum. These tasks were set to assess the ease of using the app to ensure: simplicity of the download process; ease of navigation between different sections; successful interaction on forum; successful use of electronic side-effects diary; and successful use of the prescription reminder. Women were given written instructions for the tasks and the talk aloud procedure was used to assess tasks. Women were asked to rate how user-friendly the app interface was. The women were also asked to rate their level of satisfaction regarding the usability of the app.

\section{Phase III: pilot of the app}

Eighteen women prescribed AET following breast cancer were provided with detailed instructions of how to download the app either onto their smartphone, tablet or computer and asked to use the app on their own device for a month from July 21, 2016 to August 20, 2016. During that month support was available to participants, if required, from the app developer. The electronic forum was moderated by an experienced health researcher (JB).

Semi-structured telephone interviews were conducted after the 1-month trial period to explore women's experiences and views of the app. Interviews lasted no longer than 30 minutes.

\section{Data analysis}

The focus groups and interviews in both Phase I and Phase III were digitally recorded, transcribed verbatim with consent from the participants. The data were analyzed using thematic analysis. ${ }^{26}$ Familiarization with transcripts was followed by the process of systematically coding the data to generate initial codes. After initial coding, texts were iteratively analyzed through a cyclical inductive process. Themes were identified from the initial codes, further codes were identified, leading to further new themes or combining of themes. Transcripts were revisited until no more themes could be identified. During this process, themes were merged or added until an accurate representation of the data was agreed by researchers (JB, MB, and EW).

\section{Results \\ Phase I: informing the development of the app}

Twenty women aged 46-73 years took part in this phase. Fourteen were married or living with a partner, two were divorced, one was single, and three were widowed. Education level included the following: completion of secondary $(n=8)$, completion of college or university $(n=9)$, and completion of postgraduate $(n=3)$.

Two main findings from Phase I were as follows: 1) Acceptability of the app (appropriateness of using an e app and practicality of using the app); and 2) Content suggestions (useful sections/useful information).

\section{Acceptability of the app}

Women were generally positive about the concept of an app to provide information and support while taking AET, and could see great potential for an app helping women to cope with some of the common issues that arise when taking AET.

I think having apps to provide information and support in healthcare is the way forward. It can provide specific information to specific treatment group in a paperless format - seems the sensible way [...] I would use it to help with this hormone treatment [AET].

However, not all women in the sample had a smartphone or a tablet and it became clear that to be as inclusive as possible the app would have to be available for download on multiple platforms. Furthermore, women emphasized that the app needed to be easy to download and simple to navigate between the relevant sections.

It needs to be flexible - to be downloaded on different devices. For example, I tend to use a computer - so I would want to download it onto my computer at home. I know 
I am unusual in not having a mobile phone. I expect most of the younger women would download it on their mobile phone or their tablet. It depends on the device you are used to using.

I think because of my age it can be scary to use these things. I do try though. My grandchildren help. As long as it is easy to use, obvious, intuitive.

\section{Content of the app}

Women felt a potential benefit of an app was that it could provide information that was easily accessible at the time it was needed, and could provide different levels of information so that women can choose the level appropriate to them. Women reported provision of information about AET was often at their initial treatment plan when primary treatment had been their priority. Furthermore, the level of information provided had not always been appropriate, with some women wanting detailed information of the evidence, while others wanted only summary information. One woman commented on the difficulty of getting this right:

It's a difficult one. Some women want the full picture to fully understand what they are taking. Others want a black and white sketch, but not the details. They just want to know enough. Others do not want to see the picture, they just want to get on with it without knowing too much. Catering for all is a challenge.

Most women considered peer support to be important and reported use of online support forums run by charities. However, while the women found these charity forums supportive, some women found they increased their anxiety because of the spectrum of women participating, some of whom had advanced disease. They felt a forum specifically for women taking AET after early-stage breast cancer would be useful to discuss issues and exchange tips in managing the side effects. Women also spoke of wanting to exchange information with other women about specific brands of products that had helped reduce the side effect profile, which charities and NHS information were not able to do.

It took me a long time to find out that different brands have worse side effects than others. I now go to a specific pharmacist to collect my prescription because I know he will supply the good ones. This is the sort of thing you do not find out from the health professionals. You need to discuss it with other women in the same situation as you.

Women felt the side effects they experienced were not given much attention by health professionals, and they suggested an intervention where they could record side effects to help "legitimize" discussion of the side effects with health professionals. Some women recorded their side effects in a paper diary, not only to provide evidence of their side effects at clinic appointments but also to identify patterns of and plan around the side effect profile and identify possible triggers.

My GP wasn't interested, so I took it upon myself to do something. Every day for two weeks I wrote a diary to record my side effects. It just helps you take control of the situation.

Participants also spoke of the importance of having advice around where to seek further information. Many had been overwhelmed by the amount of information provided on the Internet and suggested that the app provides links to trustworthy websites and organizations.

... there is too much out there on the Internet which is not always kosher and you can frighten yourself.

In the first focus group, women discussed their frustration of having to order repeat prescriptions every month and raised the question of including text message reminders on the app. However, involving a health professional to deliver this intervention would add an additional layer of complexity, and women suggested having a function where they could set reminders themselves instead.

... it's quite a challenge over five years - with my GP [...]

I used to get two months at a time but not every GP is as cooperative.

Following the analysis of Phase I, the research team and app developer identified the functions for the prototype app (Table 1).

The prototype app was developed to include these functions. Figure 1 shows the initial navigation page of the app. Figure 2 demonstrates the side-effects diary function.

\section{Phase II: usability of the app}

Five women aged 51-73 years who had been prescribed AET after breast cancer piloted the prototype app in terms of its usability. Women reported that its user interface provided a clear display with intuitive controls. All the set tasks were completed successfully with all participants scoring $100 \%$. Each task was completed in a timely manner, although there were delays in accessing the forum. User satisfaction was good in all tasks and few errors were recorded. Errors that did occur included problems downloading the app onto the platform chosen and contributing to the online forum. 
Table I Functions for the prototype app

\begin{tabular}{|l|l|}
\hline & Functions for prototype informed by Phase I \\
\hline I. & Evidence-based information about the treatments, side effects, and alternative AET medication, as well as coping strategies and sources of support. \\
\hline 2. & Links to current evidence, clinical trials and systematic reviews. \\
\hline 3. & $\begin{array}{l}\text { Electronic side-effects diary to record side effects of AET and their severity (mild, moderate or severe) to inform clinic appointments, or } \\
\text { for personal use, eg, identify triggers. } \\
\text { Note: common side effects include hot flushes, night sweats, anxiety, depression, joint ache, weight gain, and vaginal dryness. }\end{array}$ \\
\hline 4. & Electronic repeat prescription reminder which women could set up to remind them to get their monthly/bi-monthly prescription. \\
\hline 5. & Online forum for women to chat with other women taking AET, with a confidential messaging section for individual messages to specific people. \\
\hline 6. & Information about charities and other organizations where women can seek support. \\
\hline 7. & Links to good Internet sites and to other useful organizations. \\
\hline
\end{tabular}

Abbreviation: AET, adjuvant endocrine therapy.

\section{Phase III: pilot of the app}

Eighteen women who had been taking AET for 1-5 years participated in the pilot of the app. Table 2 below reports the sample characteristics.

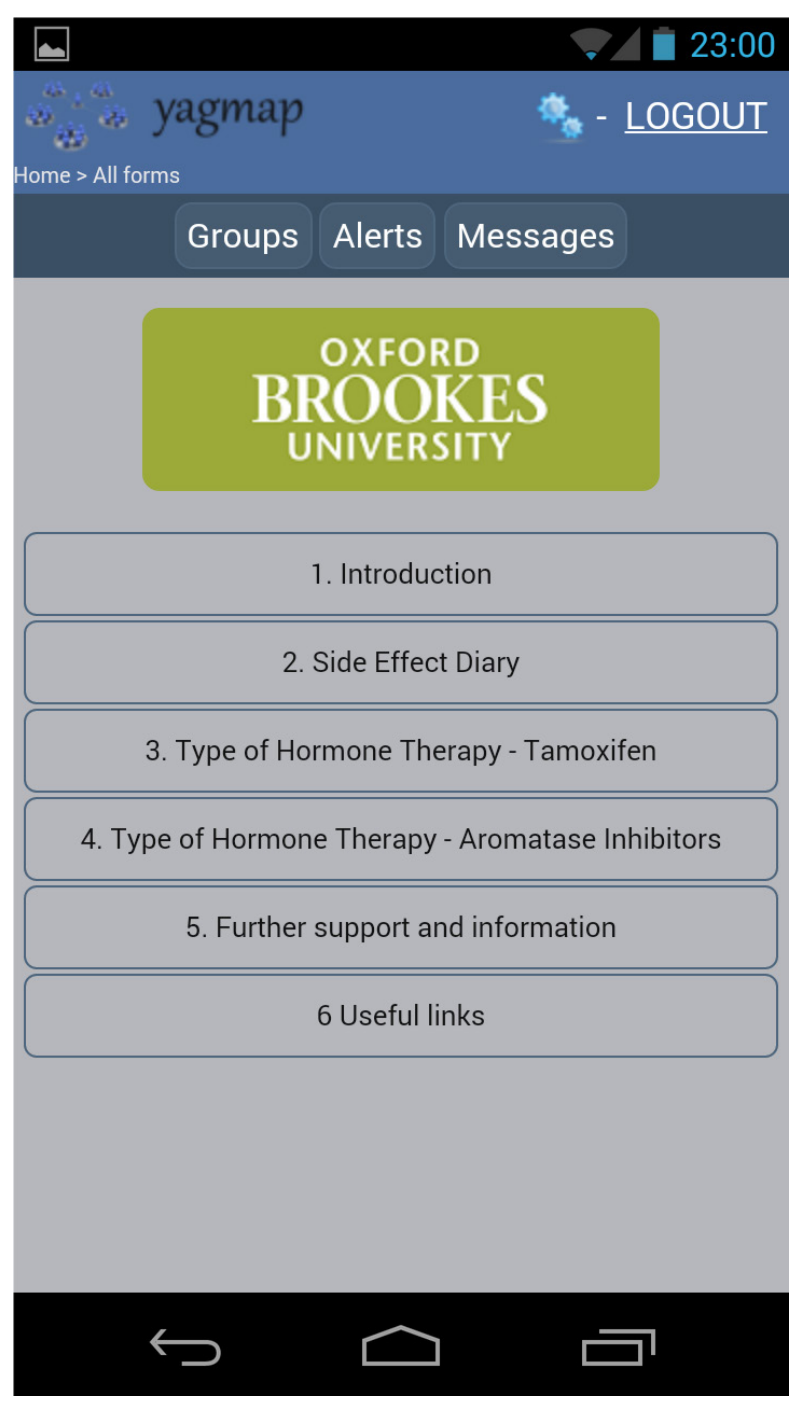

Figure I Screen shot of app home page detailing the different sections.
The data are reported by section headings of the e-health app: 1) downloading and introduction; 2) side-effects diary; and 3) information and evidence.

\section{Downloading and introduction}

Women reported that downloading and navigating between sections of the app was straightforward and that the app was

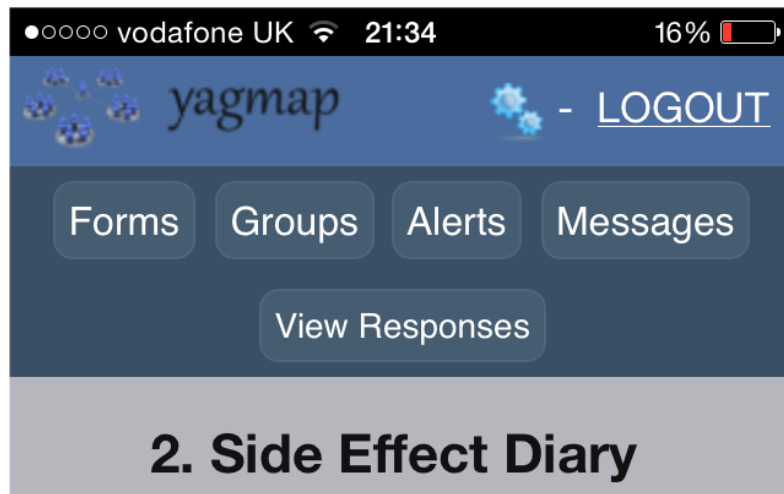

Instructions: Impact on daily life (Please select the option that best describes the impact of your side effect on your daily life)

Record any notes here (optional):

\begin{tabular}{llll}
\hline Not flush & & & \\
\hline Night sweat & & N/A & V \\
\hline
\end{tabular}

Figure 2 Screen shot of electronic side-effect diary. Groups - give access to online forum. Alerts - give access to electronic prescription reminder. Messages - for confidential messages sent between women in the forum. 
Table 2 Characteristics of the research participants in Phase III

\begin{tabular}{|l|l|}
\hline Variables & $\mathbf{n}$ \\
\hline Age (years) & \\
\hline $3 I-45$ & 2 \\
\hline $46-60$ & 7 \\
\hline $6 I-75$ & 9 \\
\hline Partner status & \\
\hline Married & 14 \\
\hline Widowed & 2 \\
\hline Single & 2 \\
\hline Treatment & \\
\hline Tamoxifen & 11 \\
\hline Aromatase inhibitors & 7 \\
\hline Reported stage of primary cancer at diagnosis & \\
\hline Stage I & 8 \\
\hline Stage 2 & 10 \\
\hline
\end{tabular}

user-friendly. Additional features suggested were a calendar, note-taking and audio-recording functions, a list of questions to ask the doctor, a glossary of terms, and links to additional resources.

Women found the introduction section helpful, guiding them through each section and providing explanations for how to maximize the use of each of the sections.

\section{Side-effects diary}

Most women used the electronic side-effects diary to record and help manage side effects and could see how it could be used to show evidence of the extent of side effects to health professionals. Several women described how it gave them a sense of control over the side effects and being able to observe good days as well as the bad days felt reassuring.

Some of the women suggested additional side effects that had not been included in this section, such as panic attacks and anxiety. Three women did not use the side-effects diary as they felt this emphasized the side effects, drawing attention to them more than they felt necessary.

The problem I have is that I get to the appointment and they start talking about the impact of the radiotherapy and all the side effects of the AET slip from my mind. If I could regularly record my side effects I could take this and show them. It would prompt a conversation about AET. [Participant 7, 54 years]

I'm trying not to study whether I have a side effect or not because it might dominate and therefore it takes over your life. [Participant 13, 68 years]

While many women completed the side-effects diary on a daily basis, some felt this was not necessary as side effects did not change much on a daily basis. Some suggested a weekly side-effects diary might be more appropriate.
I did the diary most days which I found really helpful because that was a bit - I found that the most useful I would do a diary entry most days - some of the ladies were saying what was the point of the diary - nothing changes actually some days do change for me - some are good and some not so it was good to keep a track - I found it quite reassuring as well. [Participant 9, 42 years]

\section{Information and evidence}

The information section provided details of the different types of AETs, what side effects to expect, and coping strategies for side effects. Most women found this section useful and easy to understand and commented on how reassuring they found knowing that side effects were due to AET.

Some women wanted additional information about the specific products they could use to alleviate the side effects, and how they can self-manage the side effects themselves without reliance on visiting the GP. Having personal stories of how women coped with the side effects throughout their trajectory on the drug was suggested to make it easier to relate to the information provided. Women also reported wanting more information about estrogen effects and the etiology of the cancer.

One woman commented that the information did not add much to the information provided in the leaflets she had received and on the Internet.

I was surprised how comprehensive it was - the information was good - it was easily readable - it was in everyday English so it was relatively easy to understand and follow.

[Participant 9, 42 years]

It is great to have information that makes the association between the drug and the side effect - it's reassuring that I haven't got something more serious. [Participant 3, 64 years]

\section{Online forum}

Women had mixed views of the usefulness of the online forum. Some women found the peer support comforting and reassuring, and thought it would be a good platform to swap ideas and provide advice on coping strategies.

Some women found it difficult to access the forum because the link to it was not on the home dashboard. Others said they were not brave enough to post something, while one woman expressed a preference to be private and not discuss issues on the forum.

[...] just knowing you are not alone and maybe somebody saying oh well this is what I do. [Participant 10, 31 years] 
Probably that part of the app where you can talk to other like-minded people on forum. [Participant 14, 57 years]

[...] my coping mechanism is to isolate myself and not to know anyone else's troubles - it's just not for me. [Participant 18, 74 years]

\section{Electronic prescription reminder}

Women could see the potential usefulness of being reminded to order their next prescription through the electronic alerts system. They found managing the monthly prescription requests challenging long-term and found setting up the reminder easy with the alert popping up on their phone or tablet. Several women already had their own reminder systems and said in reality they would not use the reminder system. One woman said she would not use it because she already received monthly reminder texts from her chemist.

Loved it - works a treat. I got an alert on my phone. It was really good. In fact I [...] use it for my shopping!!

[Participant 11, 63 years]

I have a really busy life so I do forget sometimes

[to order prescription] [...] I need something like this.

[Participant 12, 61 years]

\section{Useful links}

Women really appreciated being guided to reliable support organizations and Internet sites. They reported the importance of navigating those newly prescribed AET to trustworthy resources, to avoid delay in finding support. Additional charities to add to this section were suggested.

Yes that's really good. There is so much on the Internet that you often do not know where to start. A little guidance at the beginning helps you find the right support for you.

[Participant 7, 54 years]

It would have been useful to have this information at the beginning - I felt I found out about them too late. [Participant 15, 53 years]

I'm really pleased you have the Maggie, Haven and Penny Brohn plus the big ones such as Macmillan and Breast Cancer Care. [Participant 16, 67 years]

There were mixed views on providing links to the evidence on AET. Some women commented that it was important to direct women to the evidence, to ensure the information they are receiving is accurate, as many of the websites provided scary statistics. One woman, although she had not read the evidence provided, found having the section reassuring because she knew she could access it at any time. She had felt the health professionals had held back on providing too much information, so by having the evidence freely available she felt she had more trust in the efficacy of AET.

Other women reported that they did not use this section because it was too academic for them and difficult to comprehend.

[...] the weirdest thing was because it was there I felt relaxed - when you know there is evidence there and I know where to get the information - when you cannot get the information well there's no studies there's something wrong so it's a weird psychological confidence thing. [Participant 17, 65 years]

I looked at those and thought $\mathrm{Oh}$ this looks very dense I cannot deal with that - it's too much - it's not snappy - it's quite academically driven. [Participant 18, 74 years]

All but one woman said she would recommend the app to women prescribed AET. The one woman who would not recommend the app to other women reported that she did not think it would add much to the information provided by the health professionals or the current Internet sites.

\section{Discussion}

This study describes the development of an app to support women prescribed AET after breast cancer. Greater support and information around AET may help improve adherence, thus reducing the risk of recurrence and breast cancer mortality. An informative and supportive e-health app could facilitate this. The prototype of the app developed provided: evidence-based information about the different types of AETs, and the possible side effects alongside suggested coping strategies; an electronic side-effects diary to record evidence of side effects, improve awareness of side effects and to allow women to consider ways of managing the side effects; an online forum for support and exchange of suggestions of self-management; a self-setting electronic repeat prescription reminder; links to evidence of trials and systematic reviews on AET; and links to other useful organizations and Internet sites to gain further support and information. The app was positively received by most women in the pilot phase of the study, and suggestions were made to improve the individual sections. For a few women, the app would not be useful, preferring either paper copies of booklets or face-to-face discussions with health professionals and peers.

E-health resources are increasingly being used as a resource for support and information by cancer survivors. ${ }^{27,28}$ 
Previous studies have found that cancer-focused apps have the potential to enhance efforts to promote behavior change, to monitor a host of symptoms and physiological indicators of disease, and to provide real-time supportive interventions, conveniently and at low cost. ${ }^{29}$ Such interventions can provide an efficient method of providing specific support and information to the unique needs of a cancer group all on one platform. ${ }^{30-32}$ Recent studies have found that the apps successfully improve activity and quality of life $\mathrm{e}^{33-35}$ and weight reduction ${ }^{36}$ in breast cancer survivors, but no studies have reported on an app specifically aimed at educating and supporting women prescribed AET despite evidence of the need for support intervention. ${ }^{7-12}$

Apps have been used successfully to improve adherence to medication. ${ }^{37,38}$ E-health interventions such as apps provide several advantages of paper information and leaflets. Access to the information can be quicker and it is easier to keep information accurate and updated. Furthermore, the additional interactive modes such as the online forums act as an electronic bulletin board that are always available so that individuals can exchange information and mutual support while maintaining anonymity to users. Adherence apps are inexpensive, scalable, and accessible to anyone with smartphone, tablet or computer. The disadvantage of e-health interventions is the lack of access for those with low incomes, older people, and rural communities who may not have these e-health platforms.

This study provides original data on patients' views and experiences of using an innovative technology to support women prescribed AET after breast cancer. The study was a pragmatic and "real-life" exploration of the use of this technology in a realistic setting using a convenience sample. The participants used the app on their personal mobile phones, tablets or computers, making it a low cost resource. Further research is needed to develop and evaluate the app further. While the interface of the app was clear to women, the next version of the prototype could incorporate colorful icons to make it more user-friendly. Limited funds prevented the development of a more technically advanced e-health app for women after treatment for breast cancer.

\section{Conclusion}

This study describes the development and pilot of a novel patient-centered e-health app that was developed in collaboration with breast cancer patients to provide information and support for women taking AET. Further development and evaluation of the app are needed to determine whether the app supports women and improves adherence to AET following treatment for early-stage breast cancer.

\section{Ethics approval and informed consent}

Ethics approval for this study was sought and gained from Oxford Brookes University Research Ethics Committee (UREC) on November 15, 2015. The UREC registration number is 150,960 . All participants gave written informed consent to take part in the study, and it was conducted in accordance with the Declaration of Helsinki.

\section{Acknowledgments}

A huge thank you to the e-health app developer Yagmap. com, who showed amazing insight and professionalism throughout the study. We are very grateful to all the women who participated in this study. We are also grateful to the Independent Cancer Patients' Voice and Breast Cancer Care for helping us in recruitment to the study. This study was funded by the Faculty of Health and Life Sciences, Oxford Brookes University.

\section{Author contributions}

$\mathrm{JB}, \mathrm{MB}$, and $\mathrm{EW}$ made substantial contributions to the acquisition of data, analysis, and interpretation of data. They also made substantial contributions to the conception and design of the study and have been involved in drafting the article or revising it critically for important intellectual content and given final approval of the version to be published and agree to be accountable for all aspects of the work.

\section{Disclosure}

The authors report no conflicts of interest in this work.

\section{References}

1. Dowsett M, Cuzick J, Ingle J, et al. Meta-analysis of breast cancer outcomes in adjuvant trials of aromatase inhibitors versus tamoxifen. J Clin Oncol. 2010;28(3):509-518.

2. Chirgwin JH, Giobbie-Hurder A, Coates AS, et al. Treatment adherence and its impact on disease-free survival in the Breast International Group 1-98 trial of tamoxifen and letrozole, alone and in sequence. J Clin Oncol. 2016;34(21):2452-2459.

3. Makubate B, Donnan PT, Dewar JA, Thompson AM, McCowan C. Cohort study of adherence to adjuvant endocrine therapy, breast cancer recurrence and mortality. Br J Cancer. 2013;108(7):1515-1524.

4. Barron TI, Cahir C, Sharp L, Bennett K. A nested case-control study of adjuvant hormonal therapy persistence and compliance, and early breast cancer recurrence in women with stage I-III breast cancer. Br J Cancer. 2013;109(6):1513-1521.

5. Hershman DL, Shao T, Kushi LH, et al. Early discontinuation and nonadherence to adjuvant hormonal therapy are associated with increased mortality in women with breast cancer. Breast Cancer Res Treat. 2011; 126(2):529-537.

6. McCowan C, Shearer J, Donnan PT, et al. Cohort study examining tamoxifen adherence and its relationship to mortality in women with breast cancer. Br J Cancer. 2008;99(11):1763-1768.

7. Brett J, Fenlon D, Boulton M, et al. Factors associated with intentional and unintentional non-adherence to adjuvant endocrine therapy following breast cancer. Eur J Cancer Care (Engl). 2018;27(1):e12601. 
8. Brett J, Boulton M, Fenlon D, et al. Adjuvant endocrine therapy after breast cancer: a qualitative study of factors associated with adherence. Patient Prefer Adherence. 2018;12:291-300.

9. Cahir C, Dombrowski SU, Kelly CM, Kennedy MJ, Bennett K, Sharp L. Women's experiences of hormonal therapy for breast cancer: exploring influences on medication-taking behaviour. Support Care Cancer. 2015;23(11):3115-3130.

10. Van Liew JR, Christensen AJ, de Moor JS. Psychosocial factors in adjuvant hormone therapy for breast cancer: an emerging context for adherence research. J Cancer Surviv. 2014;8(3):521-531.

11. Harrow A, Dryden R, McCowan C, et al. A hard pill to swallow: a qualitative study of women's experiences of adjuvant endocrine therapy for breast cancer. BMJ Open. 2014;4(6):e005285.

12. Moon Z, Moss-Morris R, Hunter MS, Carlisle S, Hughes LD. Barriers and facilitators of adjuvant hormone therapy adherence and persistence in women with breast cancer: a systematic review. Patient Prefer Adherence. 2017;11:305-322.

13. Sparano JA, Gray RJ, Makower DF, et al. Adjuvant chemotherapy guided by a 21-gene expression assay in breast cancer. $N$ Engl J Med. 2018;379(2):111-121.

14. National Institute for Health and Care Excellence [webpage on the Internet]. Early and locally advanced breast cancer: diagnosis and management. NICE guideline [NG101] July 2018. Available from: http://www.nice.org.uk/guidance/cg80. Accessed August 3, 2018.

15. National Cancer Survivorship Initiative NCSI. 2013 Living With and Beyond Cancer: Taking Action to Improve Outcomes 2013 Mar. Available from: https://assets.publishing.service.gov.uk/government/ uploads/system/uploads/attachment_data/file/181054/9333-TSO2900664-NCSI_Report_FINAL.pdf. Accessed June 12, 2017.

16. Klasnja P, Pratt W. Healthcare in the pocket: mapping the space of mobilephone health interventions. J Biomed Inform. 2012;45(1):184-198.

17. Coulter A, Jenkinson C. European patients' views on the responsiveness of health systems and healthcare providers. Eur J Public Health. 2005; 15(4):355-360.

18. Hibbard JH, Cunningham PJ. How engaged are consumers in their health and health care, and why does it matter? Res Brief. 2008; 8:1-9.

19. Davies, NJ, Batehup L. Self-management support for cancer survivors: guidance for developing interventions. An update of the evidence. Self-Management Workstream. London: Department of Health, NCSI/ Macmillan Cancer Support; 2010.

20. Mobasheri MH, Johnston M, King D, Leff D, Thiruchelvam P, Darzi A. Smartphone breast applications - what's the evidence? Breast. 2014; 23(5):683-689.

21. Giunti G, Giunta DH, Guisado-Fernandez E, Bender JL, FernandezLuque L. A biopsy of breast cancer mobile applications: state of the practice review. Int J Med Inform. 2018;110:1-9.

22. Tiong SS, Koh ES, Delaney G, et al. An e-health strategy to facilitate care of breast cancer survivors: a pilot study. Asia Pac J Clin Oncol. 2016;12(2):181-187

23. Zhu J, Ebert L, Liu X, Chan SW. A mobile application of breast cancer e-support program versus routine care in the treatment of Chinese women with breast cancer undergoing chemotherapy: study protocol for a randomized controlled trial. BMC Cancer. 2017;17(1):291.
24. Usability.Gov [homepage on the Internet]. Usability Test Plan. Available from: https://www.Usability.Gov. Accessed May 5, 2015.

25. Nielsen J. Heuristic evaluation. In: Nielsen J, Mack RL, editors. Usability Inspection Methods. New York, NY: John Wiley \& Sons; 1994.

26. Braun V, Clarke V. Using thematic analysis in psychology. Qual Res Psychol. 2006;3(2):77-101.

27. Corbett T, Singh K, Payne L, et al. Understanding acceptability of and engagement with Web-based interventions aiming to improve quality of life in cancer survivors: a synthesis of current research. Psychooncology. 2018;27(1):22-33.

28. Chou WY, Liu B, Post S, Hesse B. Health-related Internet use among cancer survivors: data from the Health Information National Trends Survey, 2003-2008. J Cancer Surviv. 2011;5(3):263-270.

29. Bender JL, Yue RY, To MJ, Deacken L, Jadad AR. A lot of action, but not in the right direction: systematic review and content analysis of smartphone applications for the prevention, detection, and management of cancer. J Med Internet Res. 2013;15(12):e287.

30. Webb TL, Joseph J, Yardley L, Michie S. Using the internet to promote health behavior change: a systematic review and meta-analysis of the impact of theoretical basis, use of behavior change techniques, and mode of delivery on efficacy. J Med Internet Res. 2010;12(1):e4.

31. Pauwels E, Van Hoof E, Charlier C, Lechner L, De Bourdeaudhuij I. Design and process evaluation of an informative website tailored to breast cancer survivors' and intimate partners' post-treatment care needs. BMC Res Notes. 2012;5:548.

32. Gao WJ, Yuan CR. Self-management programme for cancer patients: a literature review. Int Nurs Rev. 2011;58(3):288-295.

33. Harder H, Holroyd P, Burkinshaw L, et al. A user-centred approach to developing bWell, a mobile app for arm and shoulder exercises after breast cancer treatment. J Cancer Surviv. 2017;11(6):732-742.

34. Lozano-Lozano M, Martín-Martín L, Galiano-Castillo N, et al. Integral strategy to supportive care in breast cancer survivors through occupational therapy and a m-health system: design of a randomized clinical trial. BMC Med Inform Decis Mak. 2016;16(1):150.

35. Egbring M, Far E, Roos M, et al. A mobile app to stabilize daily functional activity of breast cancer patients in collaboration with the physician: a randomized controlled clinical trial. J Med Internet Res. 2016;18(9):e238.

36. Quintiliani LM, Mann DM, Puputti M, Quinn E, Bowen DJ. Pilot and feasibility test of a mobile health-supported behavioral counseling intervention for weight management among breast cancer survivors. JMIR Cancer. 2016;2(1):e4.

37. Haase J, Farris KB, Dorsch MP. Mobile applications to improve medication adherence. Telemed J E Health. 2017;23(2):75-79.

38. Dayer L, Heldenbrand S, Anderson P, Gubbins PO, Martin BC. Smartphone medication adherence apps: potential benefits to patients and providers. J Am Pharm Assoc. 2013;53(2):172-181.
Patient Preference and Adherence

\section{Publish your work in this journal}

Patient Preference and Adherence is an international, peer-reviewed, open access journal that focuses on the growing importance of patient preference and adherence throughout the therapeutic continuum. Patient satisfaction, acceptability, quality of life, compliance, persistence and their role in developing new therapeutic modalities and compounds to optimize

\section{Dovepress}

clinical outcomes for existing disease states are major areas of interest for the journal. This journal has been accepted for indexing on PubMed Central. The manuscript management system is completely online and includes a very quick and fair peer-review system, which is all easy to use. Visit http://www. dovepress.com/testimonials.php to read real quotes from published authors. 